\title{
Pigment epithelium-derived factor facilitates NLRP3 inflammasome activation through downregulating cytidine monophosphate kinase 2: A potential treatment strategy for missed abortion
}

\author{
XI ZHANG ${ }^{1 *}, \mathrm{KUN} \mathrm{ZHANG}^{2 *}$ and $\mathrm{YI} \mathrm{ZHANG}^{1}$ \\ ${ }^{1}$ Department of Gynecology and Obstetrics, Changning Maternity and Infant Health Hospital, Shanghai 200050; \\ ${ }^{2}$ Department of Pediatrics, Sixth People's Hospital Affiliated to Shanghai Jiao Tong University, Shanghai 200233, P.R. China
}

Received August 23, 2019; Accepted December 20, 2019

DOI: $10.3892 /$ ijmm.2020.4517

\begin{abstract}
A number of conditions may underlie the occurrence of missed abortion (MA), including inflammation. Pigment epithelium-derived factor (PEDF) is a novel mediator of the inflammation-related nucleotide-binding oligomerization domain-like receptor protein 3 (NLRP3) inflammasome, which is associated with several human diseases. However, the association between MA and NLRP3 inflammasome, and whether PEDF is reduced in MA, remain unknown. In the present study, the decidua and chorion tissues of patients who had suffered a MA were examined, and a lipopolysaccharide (LPS)-induced human chorionic trophoblast HTR8/SVneo cell model was established to mimic MA in vitro. The results revealed that cytidine monophosphate kinase 2 (CMPK2) expression and NLRP3 inflammasome activation, downstream pro-IL-18 and pro-IL-1 $\beta$ expression, and IL-18 and IL-1 $\beta$ release, were all significantly increased in MA tissues or LPS-induced HTR8/SVneo cells. PEDF reversed the increase in CMPK2 expression and activation of the NLRP3 inflammasome axis and, thus, downregulated the production of mitochondrial reactive oxygen species and mitochondrial DNA release, resulting in reduced lactate dehydrogenase release, and a resultant decrease in cell viability. Recovery of CMPK2 expression abolished all the effects of PEDF, indicating that CMPK2 may be an effector downstream of PEDF. PEDF reduced CMPK2 protein levels but did not affect the mRNA levels, and treatment with the proteasomal inhibitor MG132
\end{abstract}

Correspondence to: Dr Yi Zhang, Department of Gynecology and Obstetrics, Changning Maternity and Infant Health Hospital, 773 Wuyi Road, Shanghai 200050, P.R. China

E-mail: zhang_0653@163.com

*Contributed equally

Key words: missed abortion, cytidine monophosphate kinase 2, NLRP3 inflammasome, pigment epithelium-derived factor, degradation significantly reversed this reduction in CMPK2 protein levels. Furthermore, a ubiquitination assay of immunoprecipitation demonstrated that CMPK2 was polyubiquitinated in the presence of LPS, PEDF and MG132. These results indicated that the NLRP3 inflammasome is implicated in the pathogenesis of MA, and PEDF may reduce MA through ubiquitin-dependent proteasomal degradation of CMPK2 to inhibit NLRP3 activation, which may serve as a novel strategy for preventing or reducing the risk of MA.

\section{Introduction}

Missed abortion (MA) refers to the unrecognized intrauterine death of the embryo or fetus without spontaneous expulsion in the first 20 weeks of gestation (1), and it constitutes $\sim 15 \%$ of diagnosed cases of stillbirth in the clinical setting (2). Women who suffer from MA may not be aware of a potential issue due to a lack of obvious symptoms. Surgical evacuation is the standard treatment for MA, which has been performed worldwide for the past 50 years (3). However, the high cost of surgery, as well as the complications associated with surgery and anesthesia, are the primary unresolved issues that surgeons and patients face. In addition to infection and bleeding, reduced fertility caused by surgery-induced intrauterine adhesions may be unacceptable for women with MA who wish to parent a child. Thus, researchers recommend medical or expectant management rather than surgical evacuation $(4,5)$.

The causes of MA are diverse and complex, including immune dysfunction (6), systemic infectious diseases (7), abnormal chromosome number or structure (8) and endocrine dysfunction (9). The innate immune-related nucleotide-binding oligomerization domain-like receptor protein 3 (NLRP3) inflammasome is a molecular platform that leads to excessive innate immune responses following activation by promoting the maturation of pro-inflammatory cytokines, such as IL-18 and IL-1 $\beta$ (10). Once the NLRP3 inflammasome is activated, it forms a complex with its adaptor, apoptosis-associated speck-like protein containing a caspase recruitment domain (ASC), resulting in catalysis of pro-caspase-1 and the formation of an activated caspase-1 (Casp1) p10/20 tetramer (11). The activation of Casp1 converts pro-IL-18 and pro-IL-1 $\beta$ into their 
mature forms, IL-18 and IL-1 $\beta$, which is necessary for immune regulation (12). Recent studies have demonstrated a novel regulator of the NLRP3 inflammasome, cytidine monophosphate kinase 2 (CMPK2) (13). CMPK2 is a rate-limiting enzyme that provides the deoxyribonucleotides for mitochondrial (mt)DNA synthesis, which is essential for the production of oxidized mtDNA fragments and the activation of the NLRP3 inflammasome (13). However, it is currently unknown whether the activation of the NLRP3 inflammasome-associated pathway is implicated in MA.

Pigment epithelium-derived factor (PEDF) is a multifunctional paracrine protein that is a member of the serpin superfamily (14). PEDF is widely expressed in various tissues, including the placenta and trophoblast, and exerts anti-angiogenic effects in the late stages of pregnancy $(15,16)$. Previous studies have revealed that activation of the NLRP3 inflammasome was inhibited by PEDF in ischemic cardiomyocytes through its phospholipaseA2/nutrin/patarin-like phospholipase domain-containing 2 PEDF receptor (PEDFR) (17).

In the present study, the role of PEDF in CMPK2-mediated NLRP3 inflammasome activation was determined in decidua and chorion tissues from patients with MA and in the human chorionic trophoblast HTR8/SVneo cell line. The aim was to determine the expression of CMPK2 and NLRP3 inflammasome in the decidua and chorion and in lipopolysaccharide (LPS)-induced HTR8/SVneo cells, and investigate the effects of PEDF overexpression on CMPK2 expression, NLRP3 inflammasome, $\mathrm{mt}$ reactive oxygen species (ROS) and mtDNA release.

\section{Materials and methods}

Clinical tissue collection. The protocol of the present study was approved by the Medical Ethics Committee of Shanghai Changning Maternity and Infant Health Hospital (approval no. 20170914), and all the patients included in the study provided signed informed consent. A total of 20 clinical samples including decidua and chorion tissues from MA patients, and 20 cases of samples from patients who underwent induced abortion (IA), were collected between October 2017 and September 2018 at the Shanghai Changning Maternity and Infant Health Hospital. The exclusion criteria were as follows: i) Infection with hepatitis B or C virus, immunodeficiency virus, papilloma virus, cytomegalovirus, Ureaplasma urealyticum and Chlamydia trachomatis; ii) patients who tested positive for blood group antibodies, anti-sperm, anti-cardiolipin and anti-endometrial antibodies; iii) abnormalities in chromosome number and structure; and iv) patients with any other systemic diseases or those who had received hormone treatment over the previous 3 months. Gestational age was determined based on the last menstruation and by ultrasonography. There were no significant differences in maternal age, gestational age, gravidity or parity between the MA and IA groups $(\mathrm{P}>0.05$; Table I).

Decidua and chorion tissues were washed with saline immediately after removal from the uterus. A part of each specimen was fixed with $4 \%$ paraformaldehyde at room temperature for immunohistochemical staining, and the remaining tissue was stored at $-80^{\circ} \mathrm{C}$ for protein and RNA extraction. In addition, serum was obtained from the elbow vein of MA and IA patients during fasting. The blood was left to stand for $30 \mathrm{~min}$, and then centrifuged at $2,000 \mathrm{x}$ g at $4^{\circ} \mathrm{C}$ for $15 \mathrm{~min}$. The serum was collected and stored at $-80^{\circ} \mathrm{C}$ for ELISA.

Immunohistochemistry. After the tissue collection, the samples were fixed in $4 \%$ paraformaldehyde for $24 \mathrm{~h}$ at room temperature, and then dehydrated and transparentized through graded alcohol and xylene series. The tissue was then embedded in paraffin. The paraffin-embedded decidua and chorion tissues were cut into $4-\mu \mathrm{m}$ sections, deparaffinized and rehydrated, and then blocked in $10 \%$ goat serum for $15 \mathrm{~min}$ at $37^{\circ} \mathrm{C}$. Endogenous peroxidase activity was quenched using 3\% hydrogen peroxide. Primary antibody against NLRP3 (1:100; cat. no. ab214185; Abcam) was added and incubated at $4^{\circ} \mathrm{C}$ overnight. The slides were washed with PBS for $5 \mathrm{~min}$ at room temperature three times and incubated with the secondary antibody from the streptavidin-peroxidase secondary antibody kit (cat. no. SP-9001, Beijing Zhongshan Golden Bridge Biotechnology Co., Ltd.) according to the manufacturer's instructions. After chromogenic development with 3,3'-diaminobenzidine tetrahydrochloride (cat. no. ZLI-9017, Beijing Zhongshan Golden Bridge Biotechnology Co., Ltd.), the slides were counterstained with hematoxylin for $5 \mathrm{~min}$ at room temperature, dehydrated through a graded alcohol series, and mounted with neutral gum. Brown-yellow granular cytoplasmic staining was considered as positive.

The staining intensity and positive expression area of the NLRP3 protein were evaluated by two independent pathologists. The staining intensity was classified into 4 grades as follows: $0,-; 1,+; 2,++;$ and $3,+++$. The positive expression area was also divided into 4 grades as follows: $0,<1 \% ; 1,1-33 \%$; 2, 34-66\%; and 3, 67-100\% of cells stained. The intensity and percentage scores were added up and the final NLRP3 staining score was as follows: 0 , no expression; 1 and 2 , weak expression; 3 and 4, moderate expression; and 5 and 6, strong expression.

Cell culture and treatment. Human chorionic trophoblast HTR8/SVneo cells were purchased from ATCC. The cells were plated on 60-mm dishes (Corning, Inc.) in RPMI-1640 medium (Gibco; Thermo Fisher Scientific, Inc.) supplemented with 10\% FBS (Gibco; Thermo Fisher Scientific, Inc.) and 1\% penicillin-streptomycin (Invitrogen; Thermo Fisher Scientific, Inc.), and cultured at $37^{\circ} \mathrm{C}$ in a humidified atmosphere containing $5 \% \mathrm{CO}_{2}$.

HTR8/SVneo cells were treated with LPS $(10 \mu \mathrm{g} / \mathrm{ml})$, leupeptin $(5 \mu \mathrm{g} / \mathrm{ml}), \mathrm{NH}_{4} \mathrm{Cl}(10 \mu \mathrm{mol} / \mathrm{l}), \mathrm{MG} 132(10 \mu \mathrm{mol} / \mathrm{l})$, or DMSO (all purchased from Selleck Chemicals) for $6 \mathrm{~h}$.

Lentiviral infection. Recombinant lentivirus (LV) was purchased from Shanghai GeneChem Co., Ltd. PEDF and CMPK2 overexpression plasmids were purchased from Shanghai GenePharma Co., Ltd. and packaged in 293T cells. A scrambled LV plasmid was constructed and used as a control. There was no difference in PEDF and CMPK2 protein expression between normal HTR8/SVneo cells and the scrambled control infected cells (data not shown). The concentrated titer of virus suspension was $2 \times 10^{12} \mathrm{TU} / 1$. When the confluence of HTR8/SVneo cells reached $~ 80 \%$, infection was performed using the PEDF-LV or CMPK2-LV according to the manufacturer's protocol. 
Table I. Comparison of general characteristics of patients with missed and induced abortion.

\begin{tabular}{lcrr}
\hline Characteristics & Missed abortion $(\mathrm{n}=20)$ & Induced abortion $(\mathrm{n}=20)$ & $\mathrm{P}$-value \\
\hline Maternal age (years) & $30.58 \pm 2.31$ & $29.64 \pm 2.95$ & $>0.05$ \\
Gestational age (days) & $45.76 \pm 1.88$ & $46.45 \pm 2.14$ & $>0.05$ \\
Gravidity & $2.36 \pm 0.41$ & $2.47 \pm 0.48$ & $>0.05$ \\
Parity & $0.52 \pm 0.09$ & $0.69 \pm 0.12$ & $>0.05$ \\
\hline
\end{tabular}

Values are presented as mean \pm standard deviation.

Western blotting. Total protein was extracted from decidua and chorion tissues using $100 \mathrm{mmol} / 1$ Tris- $\mathrm{HCl}, 4 \%$ SDS, $20 \%$ glycerine, $200 \mathrm{mmol} / \mathrm{l} \mathrm{DTT}$, phosphatase and protease inhibitors ( $\mathrm{pH}$ 6.8). Protein was extracted from HTR8/SVneo cells using a Cell Total Protein Extraction kit (Sangon Biotech Co., Ltd.) according to the manufacturer's protocol. Protein concentration was measured using a bicinchoninic acid (BCA) assay. Equivalent amounts of protein $(40 \mu \mathrm{g})$ were loaded on 8-12\% SDS gels and resolved using SDS-PAGE. The resolved proteins were transferred to nitrocellulose membranes (EMD Millipore). After blocking with $3 \%$ bovine serum albumin (BSA; Sangon Biotech Co., Ltd.) for $2 \mathrm{~h}$ at room temperature and washing for 3 times, the membranes were probed with antibodies specific to NLRP3 (1:1,000; cat. no. ab214185, Abcam), ASC (1:1,000; cat. no. ab155970, Abcam), Casp1 (p20) (1:1,000; cat. no. ab207802, Abcam), PEDF (1:1,000; cat. no. ab14993, Abcam), CMPK2 (1:1,000; cat. no. ab194567, Abcam), proIL-18 (1:1,000; cat. no. ab191152, Abcam), proIL-1 $\beta$ (1:1,000; cat. no. D116, Cell Signaling Technology, Inc.), and $\beta$-actin $\left(1: 1,000\right.$; cat. no. ab8226, Abcam) at $4^{\circ} \mathrm{C}$ overnight, and incubated with the relevant horseradish peroxidase (HRP)-conjugated secondary antibodies $(1: 10,000$; cat. nos. 926-32210 and 926-32221, Gene Co., Ltd.) at room temperature for $2 \mathrm{~h}$. After washing for 3 times, signals were visualized using an Odyssey Infrared Imaging system (LI-COR Biosciences). Digitized images were analyzed using ImageJ software, version d1.47 (National Institutes of Health). Protein expression levels were normalized to $\beta$-actin expression.

Immunoprecipitation. Total protein was extracted from decidua and chorion tissues using a special lysis buffer (Sangon Biotech Co., Ltd.) for immunoprecipitation. Lysates were centrifuged at $14,000 \mathrm{x} \mathrm{g}$ for $15 \mathrm{~min}$ at $4^{\circ} \mathrm{C}$, and the protein concentrations were measured using a BCA assay. Part of the supernatant was used as the input control and the rest was immunoprecipitated overnight with NLRP3 or CPMK 2 antibody at $4^{\circ} \mathrm{C}$ with gentle agitation; $\sim 4 \mu \mathrm{l}$ antibody was used per $400 \mu \mathrm{g}$ of total protein. Protein $\mathrm{A} / \mathrm{G}$ agarose beads (Santa Cruz Biotechnology, Inc.) were added to bind with the immunoprecipitants for $2 \mathrm{~h}$ with gentle agitation at room temperature. The precipitated proteins were washed three times with lysis buffer and boiled with $5 \mathrm{X}$ loading buffer, and western blotting was performed as described above. Rabbit normal immunoglobulin G (1:500; cat. no. sc-3879, Santa Cruz Biotechnology, Inc.) was used as the negative control.

Reverse transcription-quantitative $(R T-q) P C R$. Total RNA from decidua and chorion tissues and HTR8/SVneo cells was extracted using TRIzol ${ }^{\circledR}$ reagent (Invitrogen; Thermo Fisher Scientific, Inc.) according to the manufacturer's protocol. The RNA was reverse-transcribed to cDNA using the PrimeScript RT reagent kit with gDNA Eraser (Takara Bio, Inc.) at $42^{\circ} \mathrm{C}$ for $15 \mathrm{~min}$ and at $85^{\circ} \mathrm{C}$ for $5 \mathrm{~min}$. Subsequently, a total of $20 \mu \mathrm{l}$ reaction volume was made with either pro-IL-18, pro-IL-1 $\beta$, CMPK2 or $\beta$-actin-specific primers (Genewiz, Inc.), and the specific genes were amplified using a PikoReal 96 Real-Time PCR system (Thermo Fisher Scientific, Inc.) with SYBR-Green PCR Master mix (Applied Biosystems; Thermo Fisher Scientific, Inc.). The thermocycling conditions were as follows: Denaturation $\left(95^{\circ} \mathrm{C}\right.$ for $\left.30 \mathrm{sec}\right)$ and PCR reaction (40 cycles at $95^{\circ} \mathrm{C}$ for $5 \mathrm{sec}$ and $60^{\circ} \mathrm{C}$ for $30 \mathrm{sec}$ ). Relative quantitative analysis of mRNA expression levels was performed using the $2^{-\Delta \Delta \mathrm{Cq}}$ method (18) and normalized to $\beta$-actin. The sequences of the primers were as follows: Pro-IL-18 forward, 5'-GGA TCCTAACTTTGATGTAAGTTAG-3' and reverse, 5'-GAA TTCACCATGGCTGCAATACCAGAAGAAG-3'; pro-IL-1 $\beta$ forward, 5'-TGGGCCTCAAAGGAAAGA-3' and reverse, 5'-GGTGCTGATGTACCAGTT-3'; CMPK2 forward, 5'-GGC AATTATCTCGTGGCTTC-3' and reverse, 5'-GTAGCTATG GCGTAGGTGGC-3'; and $\beta$-actin forward, 5'-TAAAGACCT CTATGCCAACACAGT-3' and reverse, 5'-CACGATGGA GGGCCGGACTCATC-3'.

ELISA. The levels of IL-18 and IL-1 $\beta$ in the serum and cultured supernatants was determined using specific ELISA kits (cat. nos. BMS267-2 and BMS224-2; Lifescience) according to the manufacturer's protocol. Standard serum samples and HRP-labeled antibodies were sequentially added to the pre-coated microwells, incubated for $1 \mathrm{~h}$ at room temperature and thoroughly washed. The substrate was developed with tetramethylbenzidine, which appears as blue, then turns yellow when catalyzed by HRP. The depth of the color is positively correlated with the levels of IL-18 and IL-1 $\beta$ in the sample. The absorbance was measured at $450 \mathrm{~nm}$ using an enzyme-labeled analyzer (Varioskan LUX; Thermo Fisher Scientific, Inc.). The standard concentrations were used as the abscissa, and the relative absorbance values were plotted as the ordinate; a linear regression analysis curve was drawn.

mtROS production assay. HTR8/SVneo cells were seeded into a 48-well plate and $200 \mu \mathrm{l}$ MitoSOX (Invitrogen; Thermo Fisher Scientific, Inc.) was added for $10 \mathrm{~min}$. After washing three times with PBS, the nuclei were counterstained with Hoechst 3342 stain for $15 \mathrm{~min}$ at room temperature. The cells 

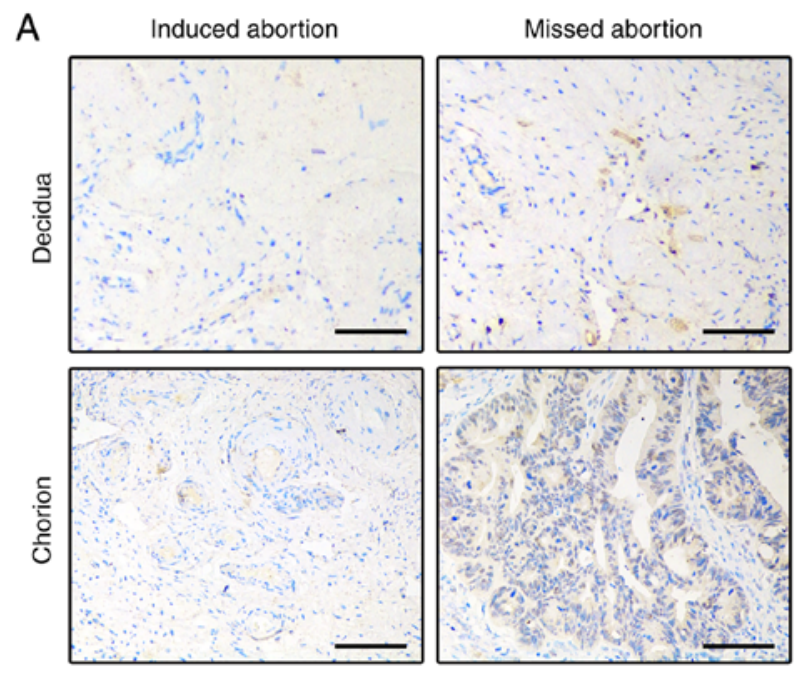

B
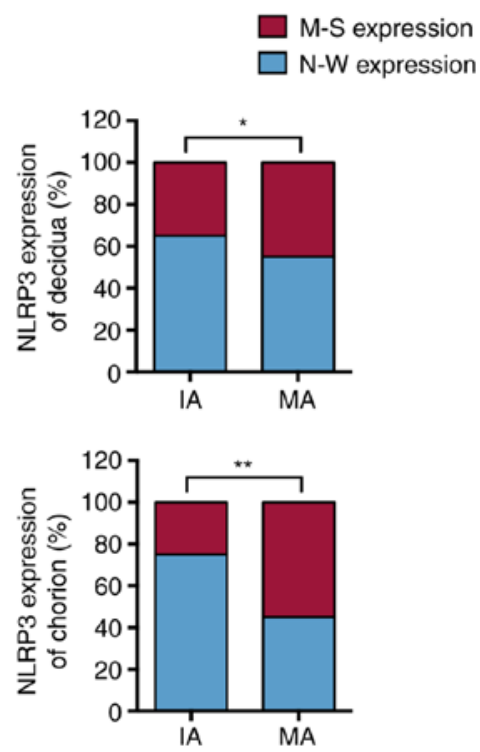

C
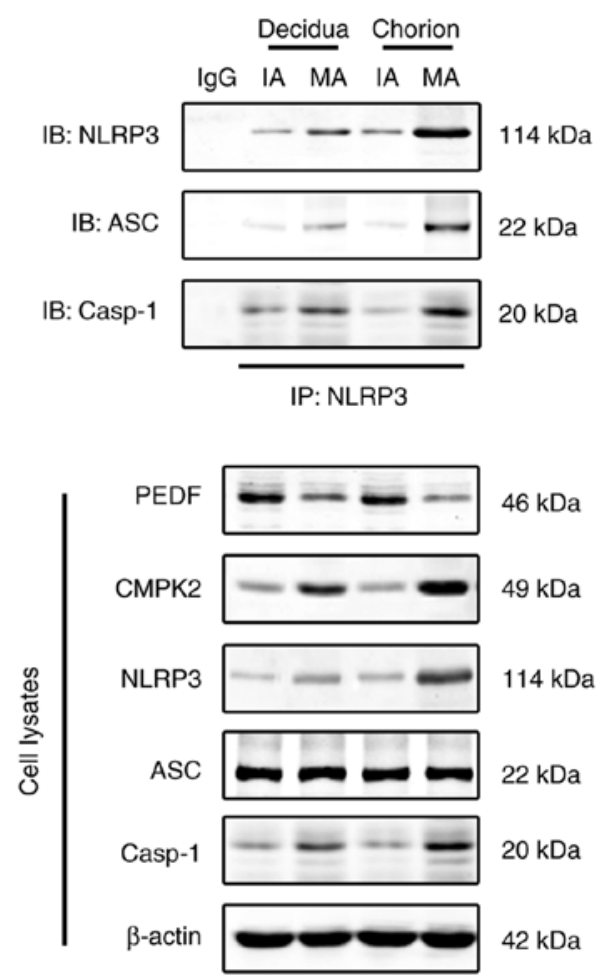

D
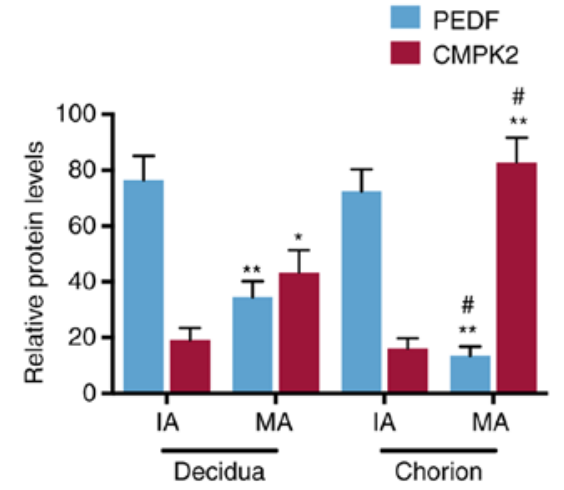

E
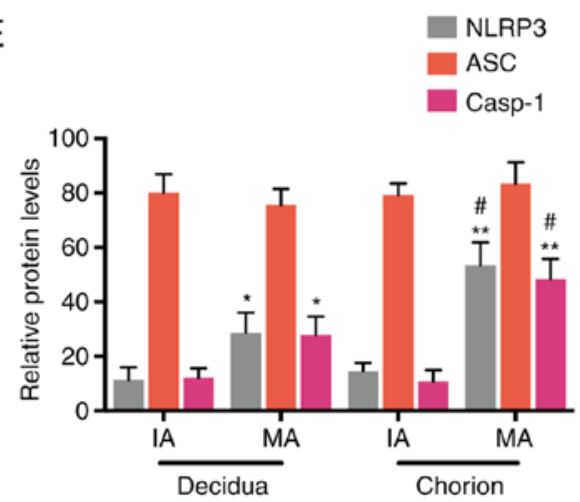

Figure 1. Activation of the NLRP3 inflammasome was observed in the decidua and chorion tissues of MA patients. (A) Immunohistochemistry staining of NLRP3 in the decidua and chorion tissues from patients who underwent IA or MA. Scale bar, $100 \mu \mathrm{m}$. (B) Ratio of moderate and strong expression to negative and weak expression of NLRP3 in decidua and chorion tissues; $n=20$. (C) Western blot of an IP of the NLRP3 inflammasome in the decidua and chorion tissues and the cell lysate input controls. Quantitative analysis of (D) PEDF and CMPK2, and (E) NLRP3, ASC and Casp1 protein expression levels; n=20. ${ }^{*} \mathrm{P}<0.05,{ }^{* *} \mathrm{P}<0.01$ vs. indicated or relative IA group; ${ }^{\#} \mathrm{P}<0.05$ vs. relative decidua tissue of MA patients. MA, missed abortion; IA, induced abortion; NLRP3, nucleotide-binding oligomerization domain-like receptor protein 3; IP, immunoprecipitation; IB, immunoblotting; PEDF, pigment epithelium-derived factor; ASC, apoptosis-associated speck-like protein containing a caspase recruitment domain; CMPK2, cytidine monophosphate kinase 2; Casp1, caspase1.

were observed using a fluorescence microscope (magnification, x100; Olympus Corporation) and the mean density of mtROS was analyzed using Image-Pro Plus, version 6.0 (Media Cybernetics).

Measurement of mtDNA. Total DNA was extracted from HTR8/SVneo cells using a DNeasy Blood \& Tissue kit (Qiagen, Inc.). The mtDNA in the cytosol was further isolated using multiple centrifugation steps, as described previously (19). The mtDNA copy number was measured using qPCR on a PikoReal 96 Real-Time PCR system (Thermo Fisher Scientific, Inc.) with SYBR-Green PCR Master mix (Applied Biosystems; Thermo Fisher Scientific, Inc.). To determine the relative mtDNA levels, the mtDNA copy number was normalized to nuclear DNA levels based on the ratio of mt cytochrome $c$ oxidase 1 (CO1) DNA vs. nuclear 18s DNA $(20,21)$. The sequences of 
A

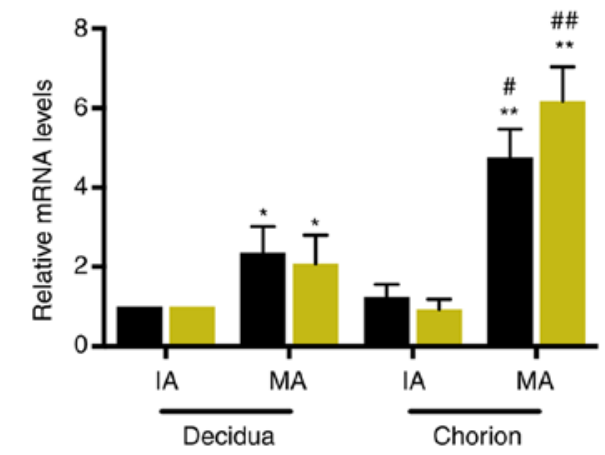

C

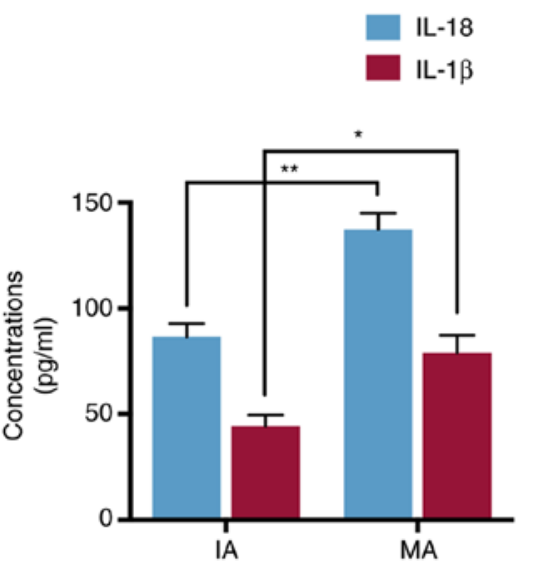

B
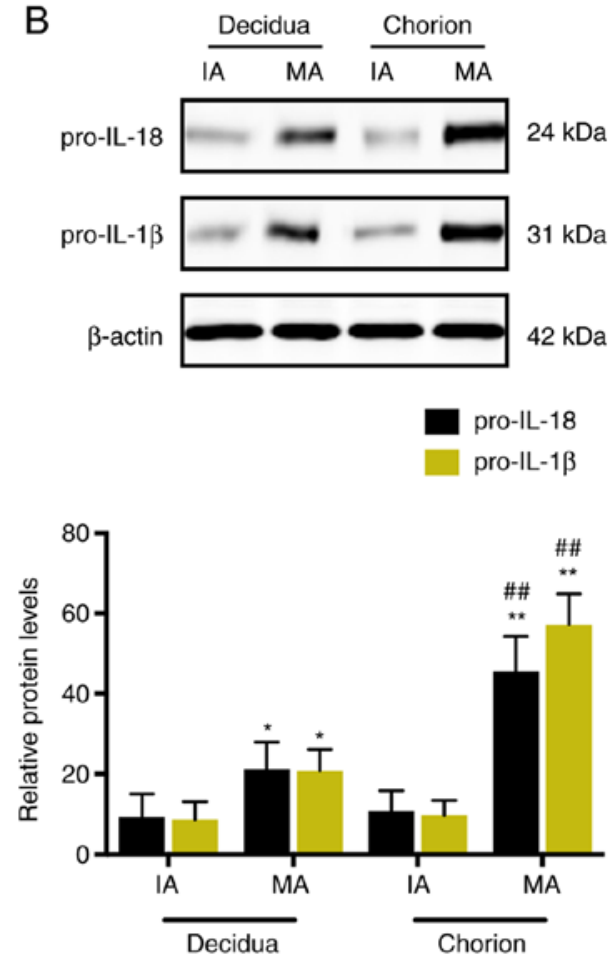

Figure 2. NLRP3 inflammasome increases downstream IL-18 and IL-1 $\beta$ levels in decidua and chorion tissues of MA patients. (A) The relative mRNA expression levels of pro-IL-18 and pro-IL-1 $\beta$ in the IA and MA groups were determined; $n=20$. (B) Western blot and densitometry analysis of pro-IL-18 and pro-IL-1 $\beta$ protein expression in IA and MA tissues; $\mathrm{n}=20$. (C) The serum concentrations of IL-18 and IL-1 $\beta$ in IA and MA tissues were analyzed using ELISA; $\mathrm{n}=20 .{ }^{*} \mathrm{P}<0.05,{ }^{* *} \mathrm{P}<0.01$ vs. relative IA group or the indicated group; ${ }^{\#} \mathrm{P}<0.05,{ }^{\# *} \mathrm{P}<0.01$ vs. relative decidua tissue of MA patients. NLRP3, nucleotide-binding oligomerization domain-like receptor protein 3; MA, missed abortion; IA, induced abortion; IL, interleukin.

the primers used were as follows: mtCO1 forward, 5'-CAG CCGTCCTACTACTTCTCTCA-3' and reverse, 5'-GATTGG GTCTCCACCTCCA-3'; and 18S forward, 5'-GACTCAACA CGGGAAACCTC-3' and reverse, 5'-AGACAAATCGCT CCACCAAC-3'.

Lactate dehydrogenase $(\mathrm{LDH})$ release and cell viability assay. HTR8/SVneo cells were seeded in 96-well plates at a concentration of $1 \times 10^{4} / \mathrm{ml}$. LDH activity in HTR8/SVneo cells released into the medium was assessed using an LDH cytotoxicity assay kit (Roche Diagnostics) according to the manufacturer's protocol. Cell viability was detected using a Cell Counting Kit- 8 according to the manufacturer's protocol (Dojindo Molecular Technologies, Inc.). Absorbance at $450 \mathrm{~nm}$ was measured using a microplate reader (BioTek Synergy 2; BioTek Instruments, Inc.). The means of the optical density measurements from 6 wells of the indicated groups were used to calculate cell viability.

Statistical analysis. Data are expressed as the mean \pm standard error of the mean. Statistical analysis was performed using SPSS version 24.0 (IBM, Corp.). Significant differences between means were analyzed using an unpaired Student's t-test, $\chi^{2}$ test, or one-way ANOVA followed by Bonferroni's post hoc test.
$\mathrm{P}<0.05$ was considered to indicate a statistically significant difference.

\section{Results}

The NLRP3 inflammasome is activated in decidua and chorion tissues of patients with MA. The expression of the NLRP3 protein was detected using immunohistochemical staining. As shown in Fig. 1A, NLRP3 expression in the decidua and chorion tissues of the MA group was higher compared with that in the IA group. In addition, the rate of moderate and strong expression of NLRP3 in the decidua of the MA group was significantly increased compared with that in the IA group (45 vs. $35 \%$, respectively; $\mathrm{P}<0.05$ ), and the rate of moderate and strong NLRP3 expression in the chorion of the MA group was further increased compared with the IA group (55 vs. $25 \%$, respectively; $\mathrm{P}<0.01$; Fig. $1 \mathrm{~B}$ ). These results suggest that NLRP3 expression is increased in patients with MA, particularly in chorion tissue.

Next, activation of the NLRP3-ASC-Casp1 (p20) inflammasome complex was determined using immunoprecipitation. As shown in Fig. 1C, NLRP3, ASC and Casp1 were strongly bound to each other in decidua and chorion tissues from the MA group. Furthermore, the protein 
A
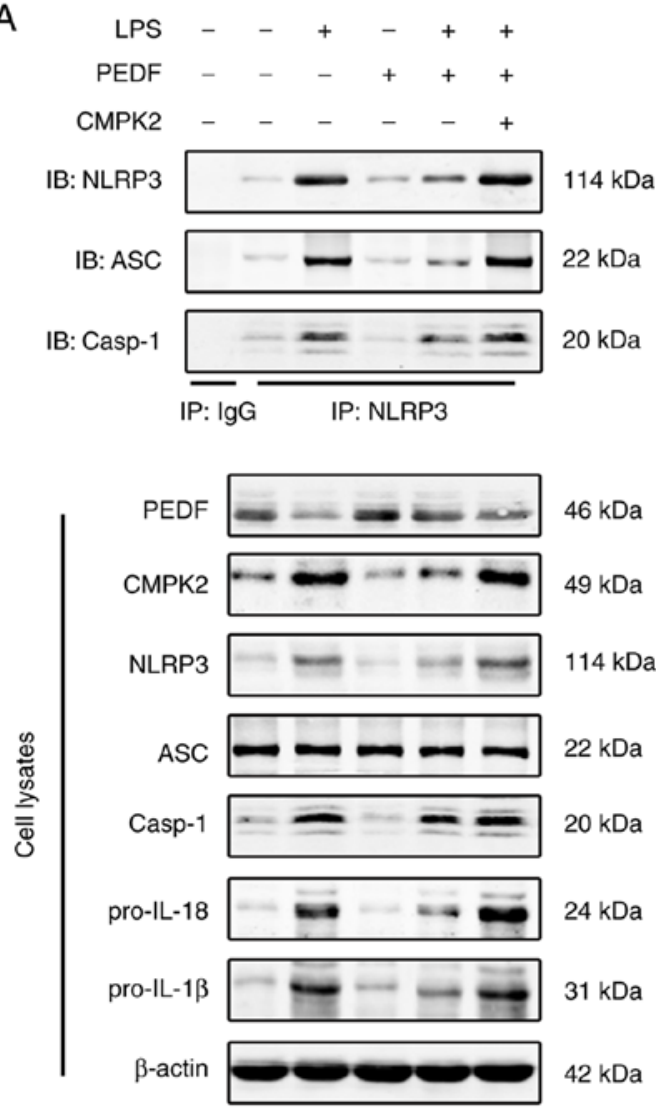

E

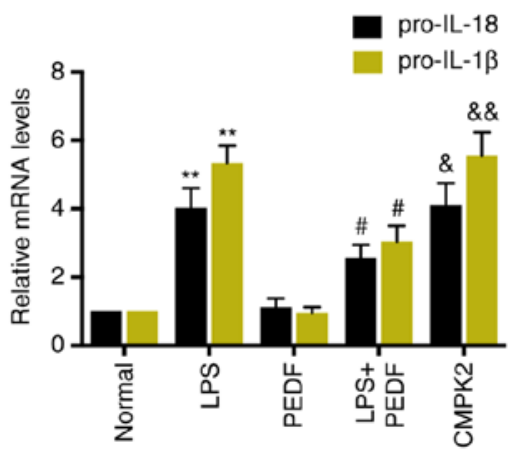

B
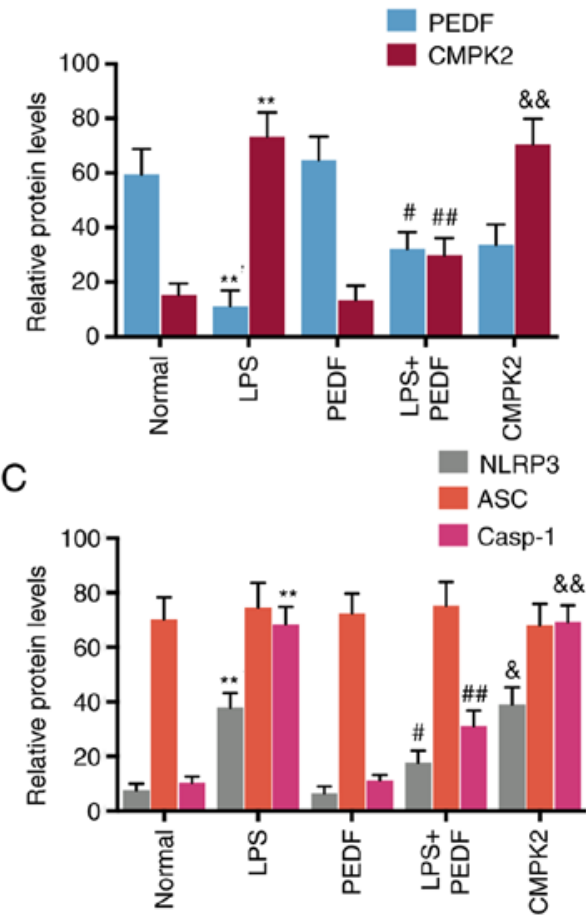

D

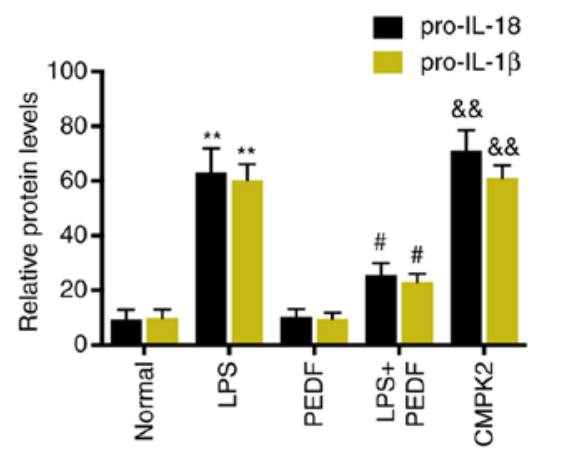

$\mathrm{F}$

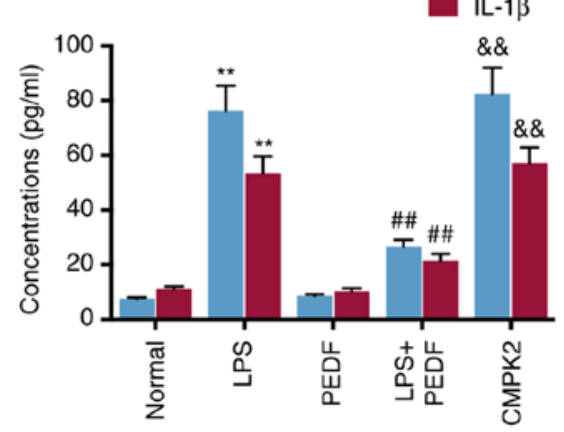

Figure 3. PEDF inhibits the activation of the NLRP3 inflammasome and reduces downstream increases in IL-18 and IL-1 1 through downregulation of CMPK2 in the HTR8/SVneo human chorionic trophoblast cell line. (A) The NLRP3 inflammasome in HTR8/SVneo cells was immunoprecipitated using an NLRP3 antibody, and the cell lysate input controls were tested by western blotting. The HTR8/SVneo cells were treated with $10 \mu \mathrm{g} / \mathrm{ml} \mathrm{LPS}$ for $6 \mathrm{~h}$. Densitometry analysis of (B) PEDF and CMPK2, (C) NLRP3, ASC and Casp1, and (D) pro-IL-18 and pro-IL-1 $\beta$ protein expression in HTR8/SVneo cells; n=5. (E) Relative mRNA levels of pro-IL-18 and pre-IL-1 $\beta$ in HTR8/SVneo cells; $n=5$. (F) The protein concentration of IL-18 and IL-1 $\beta$ in the cultured supernatants was analyzed by ELISA. $n=5 .{ }^{* *} \mathrm{P}<0.01$ vs. relative normal group; ${ }^{\sharp} \mathrm{P}<0.05$, ${ }^{\# \#} \mathrm{P}<0.01$ vs. relative LPS group; ${ }^{\circledR} \mathrm{P}<0.05$, ${ }^{\text {\&\&}} \mathrm{P}<0.01$ vs. relative LPS $+\mathrm{PEDF}$ group. $\mathrm{PEDF}$, pigment epithelium-derived factor; NLRP3, nucleotide-binding oligomerization domain-like receptor protein 3; LPS, lipopolysaccharide; ASC, apoptosis-associated speck-like protein containing a caspase recruitment domain; CMPK2, cytidine monophosphate kinase 2; Casp1, caspase 1; CMPK2 group, LPS + PEDF-LVs + CMPK2-LV; LV, lentivirus; IP, immunoprecipitation; IB, immunoblotting.

expression of CMPK2, NLRP3 and Casp1 was significantly increased in decidua $(\mathrm{P}<0.05$; Fig. 1D) and chorion tissues $(\mathrm{P}<0.01$; Fig. 1E) from the MA group compared with the IA group. The increase in the protein expression levels in MA chorion tissues was higher when compared with decidua tissues from MA patients $(\mathrm{P}<0.05)$. Conversely, PEDF expression was significantly decreased in both decidua and chorion tissues of MA patients compared with the IA group 
A
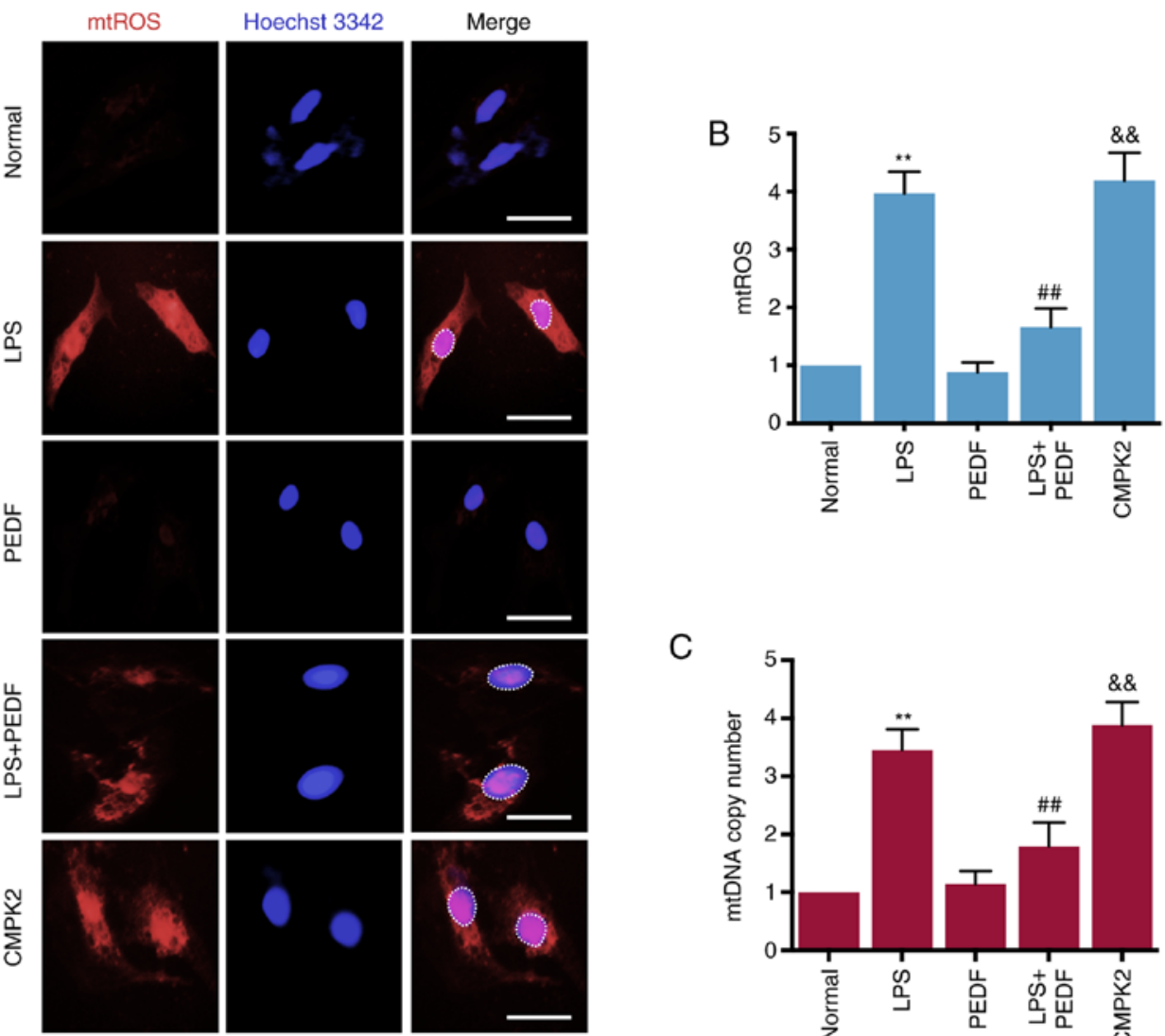

C
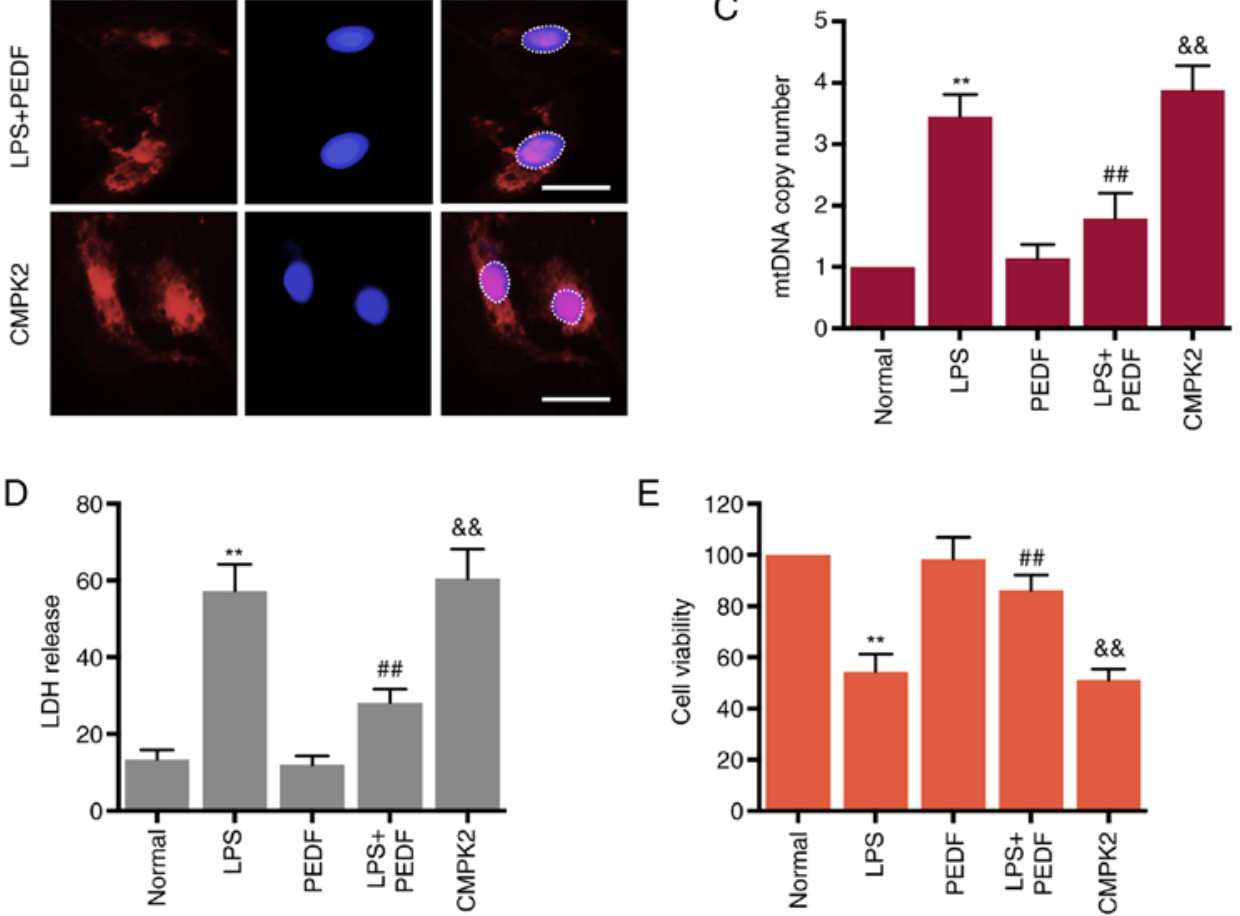

Figure 4. PEDF alleviates LPS-induced mitochondrial and cellular damage by decreasing the expression of CMPK2 in HTR8/SVneo cells. (A) Fluorescence microscopy for Mito-SOX Red-labelled ROS in cytosol of HTR8/SVneo cells. Scale bar, $100 \mu \mathrm{m}$. HTR8/SVneo cells, cells overexpressing PEDF, or cells overexpressing CMPK2, were treated with $10 \mu \mathrm{g} / \mathrm{ml}$ LPS for $6 \mathrm{~h}$. (B) Quantification of fluorescence density of mtROS in HTR8/SVneo cells. n=5. (C) Cytosolic mtDNA copy numbers were measured using by quantitative PCR; $n=5$. HTR8/SVneo (D) cell death and (E) cell viability were determined by assessing LDH release or using a Cell Counting Kit-8 assay, respectively. $n=5 .{ }^{* *} \mathrm{P}<0.01$ vs. relative normal group; ${ }^{\# \#} \mathrm{P}<0.01$ vs. relative LPS group; ${ }^{\text {\&\&}} \mathrm{P}<0.01$ vs. relative LPS + PEDF group. PEDF, pigment epithelium-derived factor; LPS, lipopolysaccharide; CMPK2, cytidine monophosphate kinase 2; mt, mitochondrial; ROS, reactive oxygen species; LDH, lactate dehydrogenase.

$(\mathrm{P}<0.01)$, and this decrease was more prominent in chorion tissues $(\mathrm{P}<0.05)$.

The NLRP3 inflammasome promotes maturation and secretion of $I L-18$ and $I L-1 \beta$ in patients with MA. As activation of the NLRP3 inflammasome modulates downstream IL-18 and IL- $1 \beta$ maturation and secretion, the mRNA and protein expression of pro-IL-18 and pro-IL-1 $\beta$, the precursor forms of IL-18 and IL-1 $\beta$, respectively, were determined. As shown in Fig. 2A and B, both the mRNA and protein expression levels of pro-IL-18 and pro-IL-1 $\beta$ were significantly increased in the decidua $(\mathrm{P}<0.05)$ and chorion $(\mathrm{P}<0.01)$ of the MA group compared with the IA group. Additionally, the levels of pro-IL-18 $(\mathrm{P}<0.01)$ and pro-IL-1 $\beta(\mathrm{P}<0.05)$ in chorion tissues were higher compared with those in the decidua in the MA group.

Subsequently, the serum concentrations of IL-18 and IL-1 $\beta$ were measured. The levels of IL-18 $(\mathrm{P}<0.05)$ and IL-1 $\beta$ $(\mathrm{P}<0.01)$ in the MA group were significantly higher compared with those in the IA group (Fig. 2C).

PEDF inhibits NLRP3 inflammasome activation and IL-18 and $I L-1 \beta$ secretion through downregulation of CMPK2 in HTR8/SVneo cells. As NLRP3 inflammasome activation was more pronounced in the chorion tissues of MA patients, LPS was used to stimulate an inflammatory response in the 
A

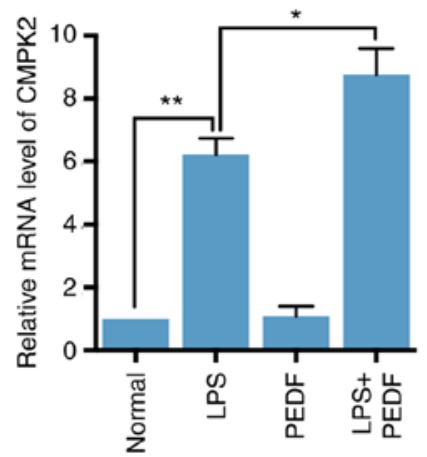

C
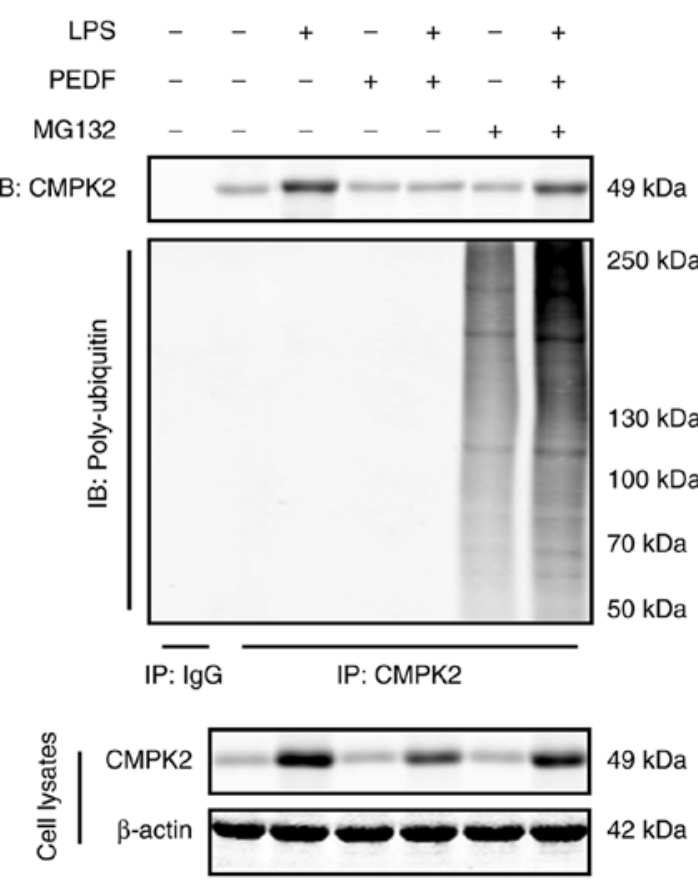

B
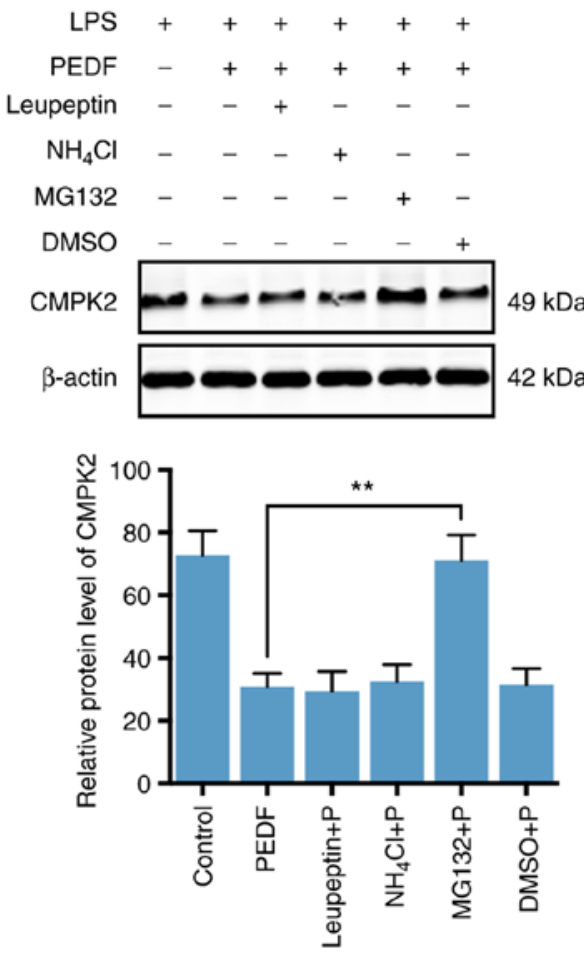

Figure 5. PEDF induces degradation of the CMPK2 protein via a ubiquitin-dependent proteasomal mechanism. (A) Relative mRNA expression levels of CMPK2 in normal, LPS-induced, PEDF-overexpressing cells, and LPS + PEDF overexpression HTR8/SVneo cells; n=5. (B) Western blot and densitometry analysis of CMPK2 in HTR8/SVneo cells treated with LPS, leupeptin general protease inhibitor, NH4Cl lysosomal inhibitor, MG132 proteasome-specific inhibitor or DMSO for $6 \mathrm{~h}$ following overexpression of PEDF; $\mathrm{n}=5$. (C) Cells were pre-treated with PEDF or MG132 and subsequently treated with LPS, and CMPK2 was immunoprecipitated; $n=5$. CMPK2 ubiquitination was determined using an anti-ubiquitin antibody. ${ }^{*} \mathrm{P}<0.05,{ }^{* *} \mathrm{P}<0.01$. $\mathrm{PEDF}, \mathrm{pigment}$ epithelium-derived factor; LPS, lipopolysaccharide; CMPK2, cytidine monophosphate kinase 2; IP, immunoprecipitation; IB, immunoblotting.

human chorionic trophoblast cell line HTR8/SVneo, in order to mimic MA in vitro $(22,23)$. LPS induction resulted in a significant increase in the binding of NLRP3, ASC and Casp1 (Fig. 3A) and increased CMPK2 (P<0.01; Fig. 3B), NLRP3 $(\mathrm{P}<0.01)$, Casp1 ( $\mathrm{P}<0.01$; Fig. 3C), and downstream pro-IL-18 and pro-IL-1 $\beta$ protein $(\mathrm{P}<0.01$; Fig. 3D) and mRNA expression $(\mathrm{P}<0.01$; Fig. $3 \mathrm{E})$ in HTR8/SVneo cells. Furthermore, the IL-18 and IL-1 $\beta$ levels in cultured supernatants were also increased following LPS induction compared with the control $(\mathrm{P}<0.01$; Fig. 3F). Overexpression of PEDF alone by infection with PEDF-LVs had no effect on the activation of the NLRP3 inflammasome. However, PEDF significantly inhibited LPS-induced activation of the NLRP3 inflammasome and increases in the aforementioned protein and mRNA expression levels.

Overexpression of CMPK2 by infection with CMPK2-LVs significantly reversed the effects of PEDF on LPS-induced activation of the NLRP3 inflammasome, suggesting that the effect of PEDF on NLRP3 inflammasome activation is associated with inhibition of CMPK 2 expression.

PEDF facilitates mitochondrial and cellular damage by reducing CMPK2 expression in LPS-induced HTR8/SVneo cells. Damage resulting from the mitochondria is primarily the result of mtROS, which alters mitochondrial structure and biogenesis through oxidation of macromolecules, such as mtDNA $(24,25)$. The cytoplasmic levels of mtROS and mtDNA were found to be associated with NLRP3 inflammasome activation $(26,27)$. Similar to the previous results, the release of mtROS and mtDNA into the cytosol was significantly increased following LPS induction compared with the control $(\mathrm{P}<0.01$; Fig. 4A-C), and overexpression of PEDF resulted in a sharp decrease in mtROS and mtDNA levels $(\mathrm{P}<0.01)$. CMPK2 overexpression significantly abolished the effects of PEDF overexpression on mtROS and mtDNA inhibition $(\mathrm{P}<0.01)$. 
Consistently, the extracellular concentration of LDH was significantly increased and cell viability was reduced in LPS-induced HTR8/SVneo cells compared with the control group $(\mathrm{P}<0.01$; Fig. 4D and E). PEDF overexpression significantly increased LPS-induced cellular damage $(\mathrm{P}<0.01)$, and CMPK2 overexpression reduced the protective effects of PEDF on LPS-induced HTR8/SVneo cells $(\mathrm{P}<0.01)$.

PEDF promotes degradation of CMPK2 through ubiquitindependent proteasomal degradation. The possible mechanism through which PEDF downregulates CMPK2 were investigated. The mRNA expression of CMPK2 was evaluated by RT-qPCR, and the results revealed that CMPK2 mRNA levels were significantly increased in the LPS-induced cells compared with the control group $(\mathrm{P}<0.01$; Fig. 5A). Overexpression of PEDF and LPS induction resulted in further increase of the CMPK 2 mRNA expression levels $(\mathrm{P}<0.05)$, suggesting a compensatory increase in mRNA expression.

CMPK2 protein levels were analyzed by treating cells with leupeptin (general protease inhibitor), $\mathrm{NH}_{4} \mathrm{Cl}$ (lysosomal inhibitor), MG132 (proteasome specific inhibitor), or DMSO for $6 \mathrm{~h}$. The results demonstrated that leupeptin and $\mathrm{NH}_{4} \mathrm{Cl}$ did not reverse the decrease in CMPK2 protein expression caused by PEDF, but CMPK2 expression was significantly increased in the MG132 + PEDF group compared with PEDF overexpression alone ( $\mathrm{P}<0.01$; Fig. $5 \mathrm{~B})$. It is well established that ubiquitination is essential for proteasome-dependent degradation (28); thus, a ubiquitination assay was performed to pull down CMPK2 through immunoprecipitation. As shown in Fig. 5C, a typical polyubiquitin protein ladder was observed in the MG132 group, demonstrating that CMPK2 was polyubiquitinated and unable to undergo proteasomal degradation (Fig. 5C). MG132 + PEDF co-treatment resulted in significant upregulation of polyubiquitinated-CMPK2 in LPS-induced HTR8/SVneo cells. These results suggest that PEDF downregulates CMPK2 expression in a ubiquitin-dependent manner.

\section{Discussion}

MA is type of unconstrained miscarriage without outside intervention. In this type of abortion, the dead fetus may be present for several weeks, and the uterus has failed to expel the dead fetus before 20 weeks' gestation (29). In recent years, the incidence of MA has increased and accounts for $15-20 \%$ of spontaneous abortions (30). There are numerous etiological factors that may explain the possible mechanisms underlying the occurrence of MA, including inflammation, immunological and endocrinological abnormalities, abnormal chromosome number or structure, inherited thrombophilia, and uterine and environmental factors (31). However, the precise mechanism of MA requires further study.

Inflammation is initiated through pathogen-associated or damage-associated molecular pattern sensing $(32,33)$. NLRP3 is a unique pattern-recognition receptor and it responds to numerous extracellular stimuli. NLRP3 exposes its pyrin domain following binding of ASC and recruits Casp1 to form a NLRP3 inflammasome complex (32-34). The assembly of the complex induces Casp1 activation to convert pro-IL-18 and pro-IL- $1 \beta$ to their mature forms, and the aberrant activation of the NLRP3 inflammasome is associated with various inflammatory and degenerative conditions (32). It was recently indicated that CMPK2 is a rate-limiting mitochondrial nucleotide kinase for de novo mtDNA synthesis, which is strongly associated with the activation of the NLRP3 inflammasome (13). However, to the best of our knowledge, the association between MA and the CMPK2-NLRP3 inflammasome has not been reported to date.

PEDF has attracted attention as an endogenous secreted protein. PEDF is a $50-\mathrm{kDa}$ protein with two primary receptors, PEDFR and laminin receptor (LR). PEDFR is widely present on the membrane of various types of cells, and when PEDF binds to PEDFR, it triggers downstream activation of phospholipase A2 enzymatic activity and lipase activity, in order to modulate inflammation and fatty acid metabolism $(35,36)$. LR is primarily expressed in endothelial cells and its functions include inhibition of cell migration, angiogenesis and tube-like network formation (37).

In the present study, an increase in NLRP3 inflammasome activation was observed in the chorion and decidua tissues of women who suffered an MA, and PEDF treatment decreased the activation of the NLRP3 inflammasome through a ubiquitin-dependent degradation pathway in an MA cell model. The upregulation in expression of NLRP 3 in MA patients was significantly higher compared with that in the IA group, and there was also an increased rate of NLRP 3 expression in the chorion compared with decidua tissue. In addition to the alterations in NLRP3 levels, the activation of the NLRP3 inflammasome, including ASC and Casp1, and CMPK2 were increased in the MA group. PEDF protein expression was abundant in the IA group, and the development of MA significantly decreased the PEDF levels, although the mechanisms underlying this decrease is unknown. Similarly, the mRNA and protein expression levels of the downstream molecules pro-IL-18 and pro-IL-1 $\beta$, and the serum IL-18 and IL-1 $\beta$ levels, were significantly increased in MA patients compared with the IA group, and the increase in chorion tissues was significantly greater compared with that in the decidua. LPS was used to induce the human chorionic trophoblast cell line HTR8/SVneo, in order to investigate the effects of PEDF MA in vitro. The expression of PEDF in LPS-treated HTR8/SVneo cells was significantly decreased compared with that in normal controls. Additionally, activation of the NLRP3 inflammasome, and the expression of CMPK2, pro-IL-18 and pro-IL- $1 \beta$, and secretion of IL-18 and IL- $1 \beta$ in HTR8/SVneo cells, were all significantly increased following LPS treatment. Furthermore, the increased mtROS production and mtDNA copy numbers in LPS-treated HTR8/SVneo cells resulted in reduced cell viability and increased release of LDH, ultimately resulting in cell death. PEDF inhibited all the effects of LPS on HTR8/SVneo cells and subsequently decreased mtROS and mtDNA release, resulting in reduced cell damage. Overexpression of CMPK2 abolished the protective effects of PEDF, suggesting that PEDF may improve MA through facilitating the activation of the NLRP3 inflammasome through modulation of CMPK2. Therefore, the role of PEDF in CMPK2 inhibition was investigated. The mRNA expression levels of CMPK2 in the LPS + PEDF group were higher compared with those in the LPS group, which may 
be attributed to the increased compensatory mRNA expression caused by the decrease in the protein expression levels. Furthermore, LPS + PEDF HTR8/SVneo cells treated with the proteasome specific inhibitor, MG132, abolished the inhibitory effect of PEDF on CMPK2, and a typical polyubiquitin protein ladder was observed in the ubiquitination assay. These results demonstrated that PEDF downregulates CMPK2 expression in a ubiquitin-dependent proteasomal degradation manner.

There were certain limitations to the present study. As there are numerous mechanisms underlying the pathogenesis of MA, and the inflammatory response is only one of these, the use of LPS-induced HTR8/SVneo cells to simulate the MA model in vitro has several limitations, and a more suitable MA cell model must be established. Furthermore, both the specific mechanism underlying the decrease in PEDF expression in MA tissues and the identification of the receptor responsible for the protective roles of exogenous PEDF on MA require further investigation. Moreover, 40 samples in present study may not be sufficient, and more samples are required in future experiments.

In conclusion, the present study demonstrated activation of the NLRP3 inflammasome in MA tissues and LPS-induced HTR8/SVneo cells; PEDF reversed the activation of the NLRP3 inflammasome in HTR8/SVneo cells, and protected cells from mitochondria-associated damage, at least partly through ubiquitin-dependent degradation of CMPK2. The findings of the present study may provide novel insight into potential improvements for the treatment of MA, and highlight the potential of PEDF-based therapies.

\section{Acknowledgements}

Not applicable.

\section{Funding}

The present study was funded by Shanghai Changning District Science and Technology Commission Research Project (grant no. CNKW2017Y14).

\section{Availability of data and materials}

The datasets generated and/or analyzed during the present study are available from the corresponding author on reasonable request.

\section{Authors' contributions}

$\mathrm{XZ}$ designed the research, performed experiments, analyzed data and wrote the manuscript. KZ designed and performed experiments and analyzed data. YZ supervised the study, managed the project and co-wrote the manuscript. All authors read and approved the final manuscript.

\section{Ethics approval and consent to participate}

The study was approved by the Medical Ethics Committee of Shanghai Changning Maternity and Infant Health Hospital, and all the patients included in the study have signed informed consents.

\section{Patient consent for publication}

The patients consented to the text and any images or videos published in the article being freely available on the internet and to the general public. The patients understand that the images, videos and text may also appear on other websites or in print, and may be translated into other languages or used for commercial purposes.

\section{Competing interests}

The authors declare that they have no competing interests.

\section{References}

1. Wu HL, Marwah S, Wang P, Wang QM and Chen XW: Misoprostol for medical treatment of missed abortion: A systematic review and network meta-analysis. Sci Rep 7: 1664, 2017.

2. Wood SL and Brain PH: Medical management of missed abortion: A randomized clinical trial. Obstet Gynecol 99: 563-566, 2002.

3. Induced abortion operations and their early sequelae. Joint study of the Royal College of General Practitioners and the Royal College of Obstetricians and Gynaecologists. J R Coll Gen Pract 35: 175-180, 1985.

4. Chia KV and Ogbo VI: Medical termination of missed abortion. J Obstet Gynaecol 22: 184-186, 2002.

5. Petrou S, Trinder J, Brocklehurst P and Smith L: Economic evaluation of alternative management methods of first-trimester miscarriage based on results from the MIST trial. BJOG 113: 879-889, 2006.

6. Cao W, Xu W, Chen T, Wang X, Wang X, Qiu J, Chen N and Mao Y: $\mathrm{CD}^{+}{ }^{+} \mathrm{CD} 25^{+} \mathrm{FoxP} 3^{+}$regulatory $\mathrm{T}$ cells and cytokines interact with estradiol in cases of missed abortion. Exp Ther Med 7: 417-422, 2014

7. Abdelshafy A, Awwad H, Abo-Gamra A, Alanwar A, Elkotb AM, Shahin M, Abd El-Razek M and Abbas AM: Sublingual vs vaginal misoprostol for completion of first trimester missed abortion: A randomised controlled trial. Eur J Contracept Reprod Health Care 24: 134-139, 2019.

8. Chatzimeletiou K, Makrydimas G and Nicolaides KH: Aneuploidy screening in a coelomic sample from a missed abortion using sequential fluorescence in situ hybridization. Fertil Steril 85: 1059 e1013-1056, 2006.

9. el-Refaey H, Hinshaw K, Henshaw R, Smith $\mathrm{N}$ and Templeton A: Medical management of missed abortion and anembryonic pregnancy. BMJ 305: 1399, 1992.

10. Schroder K and Tschopp J: The inflammasomes. Cell 140: $821-832,2010$.

11. Schroder K, Zhou R and Tschopp J: The NLRP3 inflammasome: A sensor for metabolic danger? Science 327: 296-300, 2010.

12. Slaats J, Ten Oever J, van de Veerdonk FL and Netea MG: IL-1 $\beta /$ IL-6/CRP and IL-18/ferritin: Distinct inflammatory programs in infections. PLoS Pathog 12: e1005973, 2016.

13. Zhong Z, Liang S, Sanchez-Lopez E, He F, Shalapour S, Lin XJ, Wong J, Ding S, Seki E, Schnabl B, et al: New mitochondrial DNA synthesis enables NLRP3 inflammasome activation. Nature 560: 198-203, 2018.

14. He X, Cheng R, Benyajati S and Ma JX: PEDF and its roles in physiological and pathological conditions: Implication in diabetic and hypoxia-induced angiogenic diseases. Clin Sci (Lond) 128: 805-823, 2015.

15. Stalberg C, Noda N, Polettini J, Jacobson B and Menon R: Pigment epithelial-derived factor in human fetal membranes. J Matern Fetal Neonatal Med 31: 2058-2065, 2018.

16. Loegl J, Nussbaumer E, Hiden U, Majali-Martinez A, Ghaffari-Tabrizi-Wizy N, Cvitic S, Lang I, Desoye G and Huppertz B: Pigment epithelium-derived factor (PEDF): A novel trophoblast-derived factor limiting feto-placental angiogenesis in late pregnancy. Angiogenesis 19: 373-388, 2016.

17. Zhou Z, Wang Z, Guan Q, Qiu F, Li Y, Liu Z, Zhang H, Dong H and Zhang Z: PEDF inhibits the activation of NLRP3 inflammasome in hypoxia cardiomyocytes through PEDF receptor/phospholipase A2. Int J Mol Sci 17: E2064, 2016.

18. Waters DL and Shapter FM: The polymerase chain reaction (PCR): General methods. Methods Mol Biol 1099: 65-75, 2014. 
19. Nakahira K, Haspel JA, Rathinam VA, Lee SJ, Dolinay T, Lam HC, Englert JA, Rabinovitch M, Cernadas M, Kim HP, et al: Autophagy proteins regulate innate immune responses by inhibiting the release of mitochondrial DNA mediated by the NALP3 inflammasome. Nat Immunol 12: 222-230, 2011.

20. Tal MC, Sasai M, Lee HK, Yordy B, Shadel GS and Iwasaki A: Absence of autophagy results in reactive oxygen species-dependent amplification of RLR signaling. Proc Natl Acad Sci USA 106: 2770-2775, 2009.

21. Lemasters JJ, Theruvath TP, Zhong Z and Nieminen AL: Mitochondrial calcium and the permeability transition in cell death. Biochim Biophys Acta 1787: 1395-1401, 2009.

22. Chen H, Deng X, Yang Y, Shen Y, Chao L, Wen Y and Sun Y: Expression of GRIM-19 in missed abortion and possible pathogenesis. Fertil Steril 103: 138-146.e3, 2015

23. Zhi Z, Yang W, Liu L, Jiang X and Pang L: Early missed abortion is associated with villous angiogenesis via the HIF-1alpha/VEGF signaling pathway. Arch Gynecol Obstet 298: 537-543, 2018

24. Tsutsui H, Kinugawa S and Matsushima S: Oxidative stress and mitochondrial DNA damage in heart failure. Circ J 72 (Suppl A): A31-A37, 2008.

25. Shokolenko I, Venediktova N, Bochkareva A, Wilson GL and Alexeyev MF: Oxidative stress induces degradation of mitochondrial DNA. Nucleic Acids Res 37: 2539-2548, 2009.

26. Xu Y, Shen J and Ran Z: Emerging views of mitophagy in immunity and autoimmune diseases. Autophagy: 1-15, 2019.

27. Bronner DN and O'Riordan MX: Measurement of Mitochondrial DNA Release in Response to ER Stress. Bio Protoc 6, 2016.

28. Petroski MD: The ubiquitin system, disease, and drug discovery. BMC Biochem 9 (Suppl 1): S7, 2008.

29. Griebel CP, Halvorsen J, Golemon TB and Day AA: Management of spontaneous abortion. Am Fam Physician 72: 1243-1250, 2005.
30. Chen BA and Creinin MD: Contemporary management of early pregnancy failure. Clin Obstet Gynecol 50: 67-88, 2007.

31. Fang Y, Kong B, Yang Q, Ma D and Qu X: MDM2 309 polymorphism is associated with missed abortion. Hum Reprod 24: 1346-1349, 2009.

32. Gross O, Thomas CJ, Guarda G and Tschopp J: The inflammasome: An integrated view. Immunol Rev 243: 136-151, 2011.

33. Kotas ME and Medzhitov R: Homeostasis, inflammation, and disease susceptibility. Cell 160: 816-827, 2015.

34. Lu A, Magupalli VG, Ruan J, Yin Q, Atianand MK, Vos MR, Schröder GF, Fitzgerald KA, Wu H and Egelman EH: Unified polymerization mechanism for the assembly of ASC-dependent inflammasomes. Cell 156: 1193-1206, 2014

35. Hirsch J, Johnson CL, Nelius T, Kennedy R, Riese W and Filleur S: PEDF inhibits IL8 production in prostate cancer cells through PEDF receptor/phospholipase A2 and regulation of NFkappaB and PPARgamma. Cytokine 55: 202-210, 2011.

36. Moreno-Navarrete JM, Touskova V, Sabater M, Mraz M, Drapalova J, Ortega F, Serrano M, Catalán V, Gómez-Ambrosi J, Ortiz MR, et al: Liver, but not adipose tissue PEDF gene expression is associated with insulin resistance. Int J Obes (Lond) 37: 1230-1237, 2013.

37. Matsui T, Higashimoto $\mathrm{Y}$ and Yamagishi S: Laminin receptor mediates anti-inflammatory and anti-thrombogenic effects of pigment epithelium-derived factor in myeloma cells. Biochem Biophys Res Commun 443: 847-851, 2014.

This work is licensed under a Creative Commons Attribution-NonCommercial-NoDerivatives 4.0 International (CC BY-NC-ND 4.0) License. 